\title{
Actitud ante la inmigración en estudiantes de enfermería. Valoración antes y después
} del 11-M.

\author{
Assessing attitudes of nursing students towards immigration before and \\ after 11 March.
}

Montserrat Antonín Martín y Joaquín Tomás-Sábado

Escola Universitària d'Infermeria Gimbernat. Universitat Autònoma de Barcelona

El fenómeno de la inmigración ha experimentado en nuestro país un importante cambio cuantitativo que ha llevado a triplicar el número de inmigrantes registrados en los últimos tres años. El hecho de que ciertos colectivos de inmigrantes se hayan vinculado a actos y organizaciones terroristas ha sugerido la idea de un posible deterioro de la actitud hacia los inmigrantes por parte de la población española. Los objetivos de este trabajo fueron realizar, mediante un estudio descriptivo transversal, una comparación entre las puntuaciones obtenidas en la Escala de Actitud ante la Inmigración en una muestra accidental de 204 estudiantes de enfermería que respondieron a la escala en febrero de 2003 y abril de 2004. Los resultados obtenidos permiten concluir que la actitud hacia la inmigración en los estudiantes es significativamente más positiva en el curso 2003-04 que en el curso anterior $(p<0,05)$, lo que se interpreta en términos de eficacia del énfasis docente en el principio de equidad hacia todos los usuarios, independientemente de su etnia o cultura.

Palabras clave: Inmigración, Actitud, Estudiantes de Enfermería
The phenomenon of immigration in our country has experienced an important quantitative change with the number of registered immigrants having trebled in the last three years. The fact that certain immigrant communities have been involved in terrorist attacks and organizations points to the idea of a possible deterioration of the attitudes of Spanish people toward immigrants.

The aim of this study was to compare by means of a transversal descriptive study, the results obtained in the Attitude towards Immigration Scale answered by an accidental sample of 204 nursing students in February 2003 and April 2004.

The findings suggest that the attitude toward immigration of those nursing students was significantly higher in the academic year 2003-2004 than in the previous year $(p<0,05)$, which can be accounted for by the efficiency of the educational emphasis on the equity principle for all students, regardless of ethnic and cultural variables.

Key words: immigration, attitude, nursing students

\section{INTRODUCCIÓN}

Correspondencia:

Montserrat Antonín Martín

Escola Universitària d'Infermeria Gimbernat

Avinguda de la Generalitat, s.n.

08174 Sant Cugat del Vallès (Barcelona)

E-mail: Montserrat.antonin@cesc.es
Numerosos estudios realizados en la década de los años noventa ${ }^{1,2}$ ponen de manifiesto los bajos niveles de xenofobia y/o racismo que presenta la sociedad española respecto a la inmigración en comparación 
con otros países de la Unión Europea, relacionándolo con el escaso número de inmigrantes que, tradicionalmente, se establecían en nuestro país. Sin embargo, en los últimos años el fenómeno de la inmigración ha experimentado en España un cambio cuantitativo muy importante, triplicándose el número de extranjeros inscritos en el Padrón de Habitantes en sólo tres años. En Cataluña, concretamente, la población extranjera ha pasado, de constituir el 2,9\% del total de la población en el año 2000, a superar ampliamente el $8 \%$ en la actualidad. A estas cifras habría que añadir aquellos inmigrantes que se encuentran en situaciones irregulares y que, por tanto, no figuran en las estadísticas oficiales. Es evidente que la inmigración de la última década y, sobre todo, de los últimos 5 años representa un cambio importante en la situación social, económica, cultural y laboral de nuestro país. Por otro lado, cabe destacar, la importancia de los medios de comunicación en la creación y transmisión de un "imaginario", de unos estereotipos muy definidos, en referencia a la imagen del inmigrante.

Los recientes atentados terroristas que hemos sufrido en nuestro país y la constatación de que algunos de sus autores pertenecían a colectivos de inmigrantes, ha sugerido la idea de un posible deterioro de la actitud por parte de la población autóctona hacia los inmigrantes y hacia el fenómeno de la inmigración en general, que pudiera traducirse en un aumento de los sentimientos y conductas xenófobos.

Desde una perspectiva psicológica, la actitud puede definirse como una disposición emocional, favorable o desfavorable, hacia algo ${ }^{3}$, en cuya constitución inciden elementos emocionales y componentes cognitivos, como las creencias, pensamientos $\mathrm{u}$ opiniones, que pueden acabar por manifestarse mediante conductas específicas ${ }^{4}$ positivas 0 de rechazo.

La docencia en enfermería se plantea la necesidad de formar profesionales dotados de los conocimientos básicos e instrumentos precisos para poder afrontar las incertidumbres y la complejidad de las diferentes situaciones, entendiendo que el cuidado de las personas, de todas las personas, debe incluir el respeto y la reflexión crítica sobre las ideas y los valores comprometidos en un diálogo en el que todos estamos involucrados ${ }^{5}$.

Es particularmente importante que los profesionales de la salud sean capaces de identificar y analizar sus actitudes ante los usuarios, haciendo conscientes sus propias concepciones culturales, sentimientos, juicios (y sobretodo, prejuicios) y creencias y de reconocer en qué medida inciden en su trabajo $^{6}$. Garantizar la calidad implica reconocer esta diversidad para ofrecer una respuesta adaptada y flexible ${ }^{7}$ que se traduzca en una calidad óptima de los cuidados que proporcionan.

Desde esta perspectiva, los objetivos de este trabajo han sido realizar una comparación entre las puntuaciones obtenidas en una Escala de Actitud ante la Inmigración (EAI), pasada a una muestra de estudiantes de enfermería en febrero de 2003 y abril de 2004 y analizar las posibles variaciones que pueden haberse producido.

\section{MÉTODO}

Sujetos. Se utilizó una muestra accidental formada por 204 estudiantes de enfermería. De ellos, 138 respondieron a la escala en febrero de 2003 (curso 2002-03) y los 66 restantes en abril de 2004 (curso 2003-2004). Además, 58 de los alumnos respondieron al cuestionario en ambas ocasiones.

Instrumento. Para evaluar la actitud de los estudiantes se utilizó la Escala de Actitud ante la Inmigración (EAI) desarrollada y validada por Antonín y Tomás-Sábado ${ }^{8}$. La EAI es un cuestionario autoadministrado que consta de 39 ítems con formato de respuesta tipo Likert con cuatro opciones, desde total acuerdo hasta total desacuerdo, que puntúan de cuatro a uno o de uno a cuatro, según la dirección del ítem, de manera que las posibles puntuaciones totales presentan un rango entre $39 \mathrm{y}$ 156, indicando las puntuaciones más altas actitudes más favorables hacia la inmigración. En las pruebas de validación de la escala se obtuvo un coeficiente alfa de Cronbach de 0,94, un coeficiente test-retest de 0,90 y una estructura factorial que apoya su consideración como medida básicamente unifactorial.

Procedimiento. Todos los sujetos respondieron a la escala como parte de un cuestionario anónimo que les fue administrado de forma grupal en sus propias aulas de clase. Previamente se les advirtió que la participación era voluntaria y se les garantizó la confidencialidad. El estudio se ajustó a un diseño observacional, descriptivo y transversal. Los datos fueron tabulados y analizados mediante el paquete estadístico SPSS 12.0 para Windows, realizándose comparaciones mediante prueba $\mathrm{t}$ de Student para muestras independientes, análisis de la varianza (ANOVA) y contrastes múltiples. 
Tabla 1. Puntuaciones medias de los cuatro grupos y del total

\begin{tabular}{|l|r|r|r|r|r|}
\hline & $\mathrm{N}$ & Media & $\begin{array}{c}\text { Desviación } \\
\text { típica }\end{array}$ & \multicolumn{2}{|c|}{$\begin{array}{c}\text { Intervalo de confianza } \\
\text { para la media al 95\% }\end{array}$} \\
\hline & & & & Límite inferior & Límite superior \\
\hline Primero 2002-2003 & 60 & 101,98 & 20,228 & 96,76 & 107,21 \\
Segundo 2002-2003 & 76 & 102,60 & 17,713 & 98,61 & 106,60 \\
Primero 2003-2004 & 66 & 107,98 & 23,389 & 102,24 & 113,73 \\
Segundo 2003-2004 & 58 & 109,00 & 21,959 & 103,23 & 114,77 \\
Total & 262 & 105,23 & 20,885 & 102,69 & 107,77 \\
\hline
\end{tabular}

\section{RESULTADOS}

Los 204 estudiantes participantes, 188 mujeres y 16 hombres, tenían una edad media de 22,18 (DT = 4,98 ) años, con un rango de 18 a 52.

En las respuestas al cuestionario durante el curso 2002-03, se obtuvo una media de 102,33 (DT=18,78), mientras que las respuestas en el curso 2003-04 presentan una media de 108,46 (DT=22,65). La diferencia entre ambas medias resulta estadísticamente significativa $(t=2,39 ; p<0,05)$. El grupo de alumnos que respondió en las dos ocasiones, obtuvo una media de 101,98 (DT=20,23) en el primer pase de la encuesta y 109,00 (DT=21,96) en el segundo. No obstante, en este caso, las diferencias no llegan a alcanzar significación estadística $(t=1,83 ; p=0,07)$.

La tabla 1 contiene las medias y desviaciones típicas de los cuatro grupos de alumnos que respondieron a la escala. La representación gráfica de estas puntuaciones (figura 1) permite apreciar con mayor detalle la elevación que se produce en las puntuaciones del curso 2003-04 con respecto al curso anterior.

Figura 1. Medias de los cuatro grupos

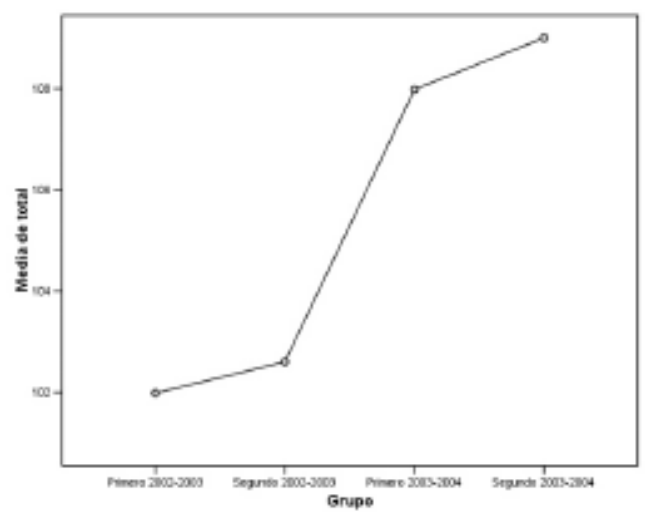

Esta tendencia se aprecia igualmente en el análisis de las puntuaciones medianas y distribución cuartil del diagrama de cajas de la figura 2 .

\section{DISCUSIÓN}

En general, los resultados obtenidos muestran un evidente aumento de las puntuaciones de la EAI en los datos obtenidos durante el curso 2003-04 respecto al curso anterior. En consecuencia, las posibles hipótesis con respecto a la posibilidad de que los recientes acontecimientos pudiesen traducirse en una actitud más negativa hacia el fenómeno de la inmigración, no presentan ningún apoyo empírico, al menos en lo que se refiere a nuestra muestra de estudiantes de enfermería, en los que parece tener más influencia el énfasis docente en el principio de equidad hacia todos los usuarios sean, o no, diversos culturalmente ${ }^{9}$.

La creciente disparidad cultural y desigualdades respecto a la salud que muestran los diversos grupos étnicos que conviven en nuestra sociedad plan-

\section{Figura 2. Distribución de las puntuaciones}

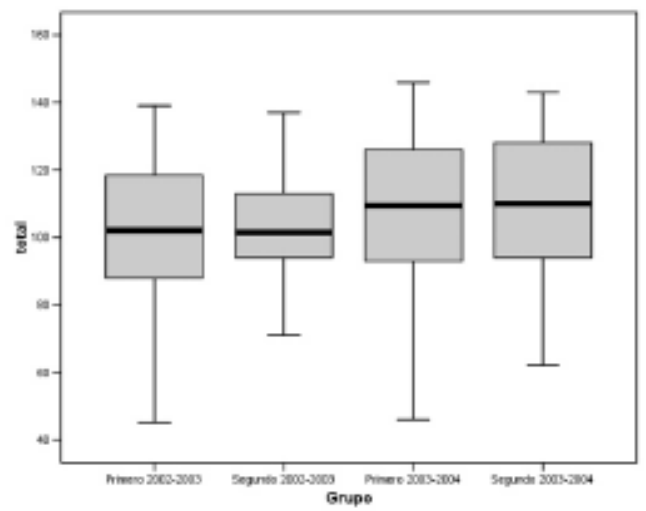


tean el reto de responder eficazmente a estas nuevas necesidades y concepciones, evidenciando, en ocasiones, la baja competencia cultural ${ }^{10}$ que muestran muchos de nuestros profesionales. Como afirman Flaskerud y Kim ${ }^{11}$, los cuidados de enfermería con la población inmigrante deben comprender no sólo la atención a la situación de enfermedad, sino también la provisión de recursos, la resolución de dilemas éticos, la superación de sentimientos xenófobos y la facilitación del pleno acceso a los servicios de salud.

Aunque los resultados de nuestro estudio permiten un cierto grado de optimismo en cuanto a la evolución de la actitud en nuestros estudiantes, lo cierto es que discrepan de los obtenidos por otros autores $^{12,13}$, que constatan un deterioro de la actitud y un aumento de los sentimientos y conductas xenófobos en estudiantes de formación preuniversitaria.

Cabe pensar que la "socialización profesional" en la que se sumergen nuestros alumnos, la "cultura de la enfermería" ${ }^{14}$ que reciben en su proceso de formación, incide en una toma de conciencia más favorable respecto a sus propias actitudes y al reto que supone conocer y cuidar a las personas con rasgos y manifestaciones culturales diferentes. De esta manera, ser conscientes de sus propias "limitaciones”, ser más críticos con sus propias actitudes, les brinda la posibilidad de reconocer en si mismos y en los demás los diversos modelos culturales ${ }^{6} \mathrm{y}$ desarrollar una mayor sensibilidad hacia el respeto y la necesidad de valorar el bagaje cultural de las personas y el compromiso con la responsabilidad de comprender la diferencia ${ }^{15}$. No debe olvidarse que el acto de cuidar presenta una dimensión ética y moral centrada en la atención benefactora de una persona hacia otra ${ }^{16}$. En consecuencia, la labor del profesional de enfermería no puede estar mediatizada por actitudes negativas hacia las diferencias étnicas y culturales de las personas a las que cuida.

En este sentido, los esfuerzos se dirigen a conseguir que los estudiantes adquieran una sensibilidad más aguda y crítica hacia los procesos culturales de las personas, independientemente de su origen, para que en su futuro como profesionales sean capaces de reconocer sus propias actitudes y gestionarlas eficazmente, modificándolas cuando sea necesario.

Es evidente, no obstante, que las características de este estudio no permiten inferir asociaciones causales en uno $u$ otro sentido y que presenta limitaciones que exigen ser cautos a la hora de interpretar los resultados e intentar extrapolarlos a la pobla- ción general. La limitación más importante está constituida por la composición de la muestra de estudiantes. Sería deseable que futuros trabajos se aplicasen al estudio de la evolución de las actitudes hacia los inmigrantes de los profesionales de enfermería, en cuanto pueden afectar a su trabajo y a la calidad de los cuidados que prestan a la sociedad.

\section{BIBLIOGRAFÍA}

1. Cea MA, Izquierdo A, Vallés, MS. Las encuestas sobre inmigración en España y Europa. Madrid: Instituto de Migraciones y Servicios Sociales; 1999.

2. Calvo Buezas T. ¿Decrece el racismo es la sociedad española? Xenofobia y hospitalidad frente a los inmigrantes. Revista Temas para el Debate 1998; 43: 47-55.

3. Morales P. Medición de actitudes en psicología y educación. San Sebastián: Ttarttalo; 1988.

4. López Pérez J. Las escalas de actitudes. En: Morales P, ed. Metodología y teoría de la psicología. Madrid: UNED; 1989.

5. Lopez D. Ética y educación para la salud. En: Sáez S, Font P, Pérez R, Marqués F, eds. Promoción y educación para la salud: conceptos, metodología, programas. Lleida: Milenio; 2001.

6. Antonín M. Antropología de la salud. En: Sáez S, Font P, Pérez R, Marqués F, eds. Promoción y educación para la salud : conceptos, metodología, programas. Lleida: Milenio; 2001.

7. Domínguez-Alcón C. Sociología del cuidado. Enferm Clínica 1999;9:174-181.

8. Antonín M, Tomás-Sábado J. La escala de actitud ante la inmigración para enfermería: Validación preliminar. Enferm Cíentífica 2004; (262-263):77-82.

9. Niederhauser VP. Health care of immigrant children: incorporating culture into practice. Pediatr Nurs 1989; 15: 569-574.

10. Allué X. La competencia cultural de los médicos de asistencia primaria. En: XV Congreso de la SVMFIC; 2003.

11. Flaskerud JH, Kim S. Health problems of Asian and Latino immigrants. Nurs Clin North Am 1999; 34: 359-380.

12. Calvo Buezas T. Racismo y escuela. Cuadernos de Pedagogía 1994; 228: 75-78.

13. Castiello JM, Nicieza J. Diversidad cultural e inmigración; reflexiones para una educación antirracista. Signos 1995; 15 : 62-69.

14. Spector R. Las Culturas de la Salud. Madrid: Prentice Hall; 2003.

15. Comelles JM. Migracions i Vulnerabilitat. L'adaptació de les institucions i les professions sanitàries a la diversitat social i cultural. En: XIII Jornades de la Societat de Salut Pública de Catalunya i de Balears; 2004.

16. Cortis JD, Kendrick K. Nursing ethics, caring and culture. Nurs Ethics 2003; 10: 77-88. 